\title{
How long does orthodontic treatment last?
}

\section{The long-term benefits of orthodontic treatment}

\section{R. A. Linklater and N. A. Fox Br Dent J 2002; 192: 583-587}

\section{Objective}

This study aimed to evaluate post-treatment changes at least five years out of retention in a cohort of 100 consecutively started patients treated by a postgraduate student in a UK orthodontic department.

\section{Design}

Analysis of study models using recognised occlusal indices.

Setting

Patients initially treated at Newcastle Dental Hospital were recalled for record collection.

\section{Materials and methods}

Study models and clinical photographs were taken for each patient attending for post-retention records. Weighted Peer Assessment Rating (PAR) Index and Index of Orthodontic Treatment Need (IOTN) scores were recorded for cases at the beginning, end and at least 5 years post-retention.

\section{Main outcome measures}

Post-treatment changes were evaluated using PAR Index and IOTN to evaluate extent and likelihood of change and to compare treatment modalities.

\section{Results}

Seventy-eight patients attended for post-retention records. Overall PAR reduction immediately post-treatment was $68.6 \%$ and at a mean of 6.5 years post-retention was 55.5\%. Dual arch fixed appliance treatments achieved the greatest reduction in PAR score, and maintained the reduction beyond the retention period better than other treatment modalities. Eighty-four per cent of the cohort still had some demonstrable improvement after a mean of 6.5 years post-retention. There was a residual need for treatment in $8 \%$ of cases on aesthetic grounds and in $24 \%$ of cases on dental health grounds.

\section{Conclusions}

Occlusal deterioration after orthodontic treatment is almost universal. Dual arch fixed appliance treatment provides the best posttreatment and post-retention result.

\section{COMMENT}

In the current climate of increased scrutiny of the training that healthcare specialists receive and the outcomes they achieve, this paper is timely.

The authors report the results of a long-term follow-up study of 78 patients who were treated by a trainee orthodontist. Uniquely the outcome of the orthodontic treatment was assessed at least five years out of retention.

When the degree of deviation from perfect occlusion and alignment were measured, using the Peer Assessment Rating Index (PAR), over $90 \%$ of the patients were rated as improved or greatly improved at the end of their orthodontic treatment. The fact that these outcomes were achieved by a trainee rather than by an experienced orthodontist is remarkable and underlines the strengths of the UK's training programmes for orthodontists, which are much admired around the world.

The long-term results provide reassurance that the vast majority of patients who receive orthodontic treatment will maintain their improvement in occlusion and dental alignment. Five years out of retention the PAR ratings revealed that $84 \%$ of the patients were still improved or greatly improved compared with their original malocclusion.

It is now clear that the health gain from orthodontic treatment should be more properly considered in psychosocial terms rather than from the perspective of oral disease prevention. In this study a surrogate measure of psychosocial impairment was used, the 10grade Aesthetic Component of the Index of Orthodontic Treatment Need. Before treatment began $80 \%$ of the patients were in the most severe aesthetic impairment category, and five years after treatment only $8 \%$ of the patients were in this category. Although this is a complex area which remains largely unexplored, it seems reasonable to assume that the patients treated in this sample will have derived significant psychosocial benefit from their orthodontic treatment.

Perhaps the most important lesson to be learnt from this paper is that serious study of healthcare outcomes requires diligent record keeping along with objective and valid measurement techniques. All these features are present in this investigation.

Donald Burden

Department of Orthodontics, Queen's University, Belfast

\section{IN BRIEF}

Occlusal deterioration occurs in the period that follows orthodontic retention.

1 Dual arch fixed appliance treatment achieves the best posttreatment results.

1 Dual arch fixed appliance treatment maintains the best postretention results.

1 Eighty-four per cent of patients in this cohort still had demonstrable benefit from orthodontic treatment after a mean of 6.5 years postretention. 